\title{
A characterization of Lie algebras of skew-symmetric elements.
}

\author{
Alexander N. Grishkov* \\ Departamento de Matemática, Universidade de São Paulo, \\ Caixa Postal 66281, São Paulo-SP, 05311-970, Brasil \\ Ivan P. Shestakov ${ }^{\dagger}$ \\ Departamento de Matemática, Universidade de São Paulo \\ and Sobolev Institute of Mathematics, Novosibirsk, 630090, Russia
}

\begin{abstract}
A characterization of Lie algebras of skew-symmetric elements of associative algebras with involution is obtained. It is proved that a Lie algebra $L$ is isomorphic to a Lie algebra of skew-symmetric elements of an associative algebra with involution if and only if $L$ admits an additional (Jordan) trilinear operation $\{x, y, z\}$ that satisfies the identities

$$
\begin{aligned}
\{x, y, z\} & =\{z, y, x\} \\
{[[x, y], z] } & =\{x, y, z\}-\{y, x, z\} \\
{[\{x, y, z\}, t] } & =\{[x, t], y, z\}+\{x,[y, t], z\}+\{x, y,[z, t]\}, \\
\{\{x, y, z\}, t, v\} & =\{\{x, t, v\}, y, z\}-\{x,\{y, v, t\}, z\}+\{x, y,\{z, t, v\}\},
\end{aligned}
$$
\end{abstract}

where $[x, y]$ stands for the multiplication in $L$.

Let $(A, *)$ be an associative algebra with an involution $*$. It is well-known that the set $H(A, *)=\left\{a \in A \mid a^{*}=a\right\}$ of symmetric elements of $A$ is closed with respect to the Jordan product $a \circ b=1 / 2(a b+b a)$ and forms a Jordan algebra with respect to this product, while the set $K(A, *)=\left\{a \in A \mid a^{*}=-a\right\}$ of skew-symmetric elements of $A$ forms a Lie algebra with respect to the commutator product $[a, b]=a b-b a$.

*Supported by CAPES, Brazil, grant 0469/02-5.

†Supported by CAPES, Brazil, grant 0461/02-4. 
In 1957 P.Cohn [2] gave a characterization of Jordan algebras of symmetric elements. He proved that a Jordan algebra $J$ is isomorphic to a Jordan algebra of type $H(A, *)$ for a certain associative algebra with involution $(A, *)$ if and only if $J$ admits an additional quadrilinear operation $[x, y, z, t]$ that satisfies some identities involving the multiplication in $J$.

Here we prove an analogue of Cohn's result for Lie algebras of skew-symmetric elements. Namely, we prove that a Lie algebra $L$ is isomorphic to a Lie algebra of type $K(A, *)$ for a certain associative algebra with involution $(A, *)$ if and only if $L$ admits a trilinear product $\{x, y, z\}$ such that, with respect to this triple product and the original Lie multiplication $[x, y], L$ forms a so called Lie-Jordan algebra (see [1]).

Let us recall the definition of Lie-Jordan algebra. A vector space $L$ is called a Lie-Jordan algebra if $L$ has bilinear operation [,] and trilinear operation $\{,$,$\} such that the following$ identities hold:

$$
\begin{aligned}
\{x, y, z\} & =\{z, y, x\} \\
{[[x, y], z] } & =\{x, y, z\}-\{y, x, z\}, \\
{[\{x, y, z\}, t] } & =\{[x, t], y, z\}+\{x,[y, t], z\}+\{x, y,[z, t]\}, \\
\{\{x, y, z\}, t, v\} & =\{\{x, t, v\}, y, z\}-\{x,\{y, v, t\}, z\}+\{x, y,\{z, t, v\}\} .
\end{aligned}
$$

It is easy to see that every Lie-Jordan algebra is a Lie algebra with respect to the operation [,] and a Jordan triple system with respect to the ternary operation $\{,$,$\} . Furthermore,$ every associative algebra $A$ forms a Lie-Jordan algebra $A^{+}$with respect to the commutator product $[x, y]$ and the triple Jordan product $\{x, y, z\}=x y z+z y x$. The main result of our previous paper [1] claims that every Lie-Jordan algebra is special, that is, isomorphic to a subalgebra of the algebra $A^{+}$for a certain associative algebra $A$.

Now, if $A$ has an involution $*$ then evidently the space $K(A, *)$ is closed with respect to the both commutator and triple Jordan product and hence is a subalgebra of the Lie-Jordan algebra $A^{+}$.

We will prove that, conversely, every Lie-Jordan algebra is isomorphic to an algebra of type $K(A, *)$. 
Theorem 1 Let $L$ be a Lie algebra over a field $k$ of characteristic $\neq 2$. Then $L$ is isomorphic to a Lie algebra $K(A, *)$ for a certain associative algebra with involution $(A, *)$ if and only if $L$ admits a trilinear operation $\{,$,$\} such that L$ is a Lie-Jordan algebra.

Proof. Let $L$ be a Lie-Jordan algebra; then an associative algebra $U(L)$ is said to be a universal enveloping algebra for $L$ if there exists a homomorphism $\alpha_{L}: L \longrightarrow U(L)^{+}$ such that for any associative algebra $A$ and a homomorphism $\beta: L \longrightarrow A^{+}$there exists a homomorphism $\pi$ of associative algebras $\pi: U(L) \longrightarrow A$ such that $\beta=\alpha_{L} \circ \pi$. In other words, there is a bijection

$$
\operatorname{Hom}_{\underline{\text { Lie-Jord }}}\left(L, A^{+}\right) \longrightarrow \operatorname{Hom}_{\underline{A s s}}(U(L), A)
$$

which is functorial on the variables $L$ and $A$.

The existence of a universal enveloping algebra $U(L)$ for a given Lie-Jordan algebra $L$ is obvious. It is isomorphic to the quotient algebra of the tensor algebra $T(L)$ by the ideal $I$ generated by all the elements

$$
a \otimes b-b \otimes a-[a, b], \quad a \otimes b \otimes c+c \otimes b \otimes a-\{a, b, c\}, a, b, c \in L
$$

with the universal homomorphism $\alpha_{L}: a \longrightarrow a+I$. By [1, Theorem 1], we have ker $\alpha_{L}=0$, hence we can identify $L$ with its image $\alpha_{L}(L)$ and assume that $L$ is a subalgebra of $U(L)^{+}$.

Define on the tensor algebra $T(L)$ an involution $*$ by setting $l^{*}=-l$ for every $l \in L$. For any $a, b, c \in L$ we have

$$
\begin{aligned}
(a \otimes b-b \otimes a-[a, b])^{*} & =b^{*} \otimes a^{*}-a^{*} \otimes b^{*}-([a, b])^{*}=b \otimes a-a \otimes b+[a, b] \\
& =-(a \otimes b-b \otimes a-[a, b]) \in I ; \\
(a \otimes b \otimes c+c \otimes b \otimes a-\{a, b, c\})^{*} & =c^{*} \otimes b^{*} \otimes a^{*}+a^{*} \otimes b^{*} \otimes c^{*}-\{a, b, c\}^{*} \\
& =-(a \otimes b \otimes c+c \otimes b \otimes a-\{a, b, c\}) \in I .
\end{aligned}
$$

Hence the ideal $I$ is invariant with respect to the involution $*$, and so this involution induces an involution on the quotient algebra $U(L)=T(L) / I$. We will denote the induced involution also by $*$. 
Let us prove that $L=K(U(L), *)$. It is clear that $L \subseteq K(U(L), *)$. In order to prove the inverse inclusion, consider the structure of $U(L)$. Since $L$ generates $U(L)$, we have

$$
U(L)=L+L L+L L L+\cdots
$$

For any $a, b, c \in L$ we have

$$
a \otimes b \otimes c-1 / 2(\{a, b, c\}+[b, c] \otimes a+b \otimes[a, c]+[a, b] \otimes c) \in I,
$$

which implies that $U(L)=L+L L$. Moreover, for any $a, b \in L$ we have

$$
a b=a \circ b+1 / 2[a, b]
$$

and since $[a, b] \in L$, this yields that $U(L)=L+L \circ L$. Evidently, $L \circ L \subseteq H(U(L)$, *), so we finally have

$$
K(U(L), *)=L, \quad H(U(L), *)=L \circ L
$$

This proves the theorem.

\section{References}

[1] A.N.Grishkov, I.P.Shestakov, Speciality of Lie-Jordan Algebras, Journal of Algebra 237, (2001) 621-636.

[2] P. M. Cohn, Two embedding theorems for Jordan algebras, Proc. London Math. Soc. (3) 9 (1959) 503-524. 